\title{
A bound on the number of points of a curve in projective space over a finite field
}

\author{
Masaaki Homma * \\ Department of Mathematics, Kanagawa University \\ Yokohama 221-8686, Japan \\ homma@kanagawa-u.ac.jp
}

\begin{abstract}
For a nondegenerate irreducible curve $C$ of degree $d$ in $\mathbb{P}^{r}$ over $\mathbb{F}_{q}$ with $r \geq 3$, we prove that the number $N_{q}(C)$ of $\mathbb{F}_{q}$-points of $C$ satisfies the inequality $N_{q}(C) \leq(d-1) q+1$, which is known as Sziklai's bound if $r=2$.

Key Words: Curve, Rational Point, Finite field, Sziklai's bound $M S C: 14 \mathrm{G} 15,11 \mathrm{G} 20,14 \mathrm{H} 25$
\end{abstract}

\section{Introduction}

In the series of papers $3,4,5$, , we proved that for any plane curve $C$ of degree $d$ over $\mathbb{F}_{q}$ without $\mathbb{F}_{q}$-linear components, the number $N_{q}(C)$ of $\mathbb{F}_{q}$-points of $C$ is bounded by

$$
N_{q}(C) \leq(d-1) q+1
$$

except for the curve over $\mathbb{F}_{4}$ defined by

$$
K:(X+Y+Z)^{4}+(X Y+Y Z+Z X)^{2}+X Y Z(X+Y+Z)=0 .
$$

Indeed, $N_{4}(K)=14$.

The bound (1) was originally conjectured by Sziklai [8], and he found that some curves actually achieve this bound.

The question we are interesting in is whether the bound (1) is valid for curves in higher dimensional projective space.

Theorem 1.1 Let $C$ be an absolutely irreducible curve of degree $d$ defined over $\mathbb{F}_{q}$ in $\mathbb{P}^{r}$ with $r \geq 3$, which is not contained in any planes. Then

$$
N_{q}(C) \leq(d-1) q+1 .
$$

*Partially supported by Grant-in-Aid for Scientific Research (21540051), JSPS. 
The main ingredient of our proof of this theorem is the order-sequence 1 of a projective curve like Stöhr-Voloch theory [7], however, our bound does not involve the genus of the curve.

As a corollary of this theorem, we have the following fact.

Corollary 1.2 Let $C$ be a curve, which may have several components, of degree $d$ in $\mathbb{P}^{r}$ over $\mathbb{F}_{q}$ without $\mathbb{F}_{q}$-linear components. In addition, when $q=d=4, C$ is not a planar curve which is isomorphic to $K$ over $\mathbb{F}_{4}$. Then

$$
N_{q}(C) \leq(d-1) q+1 .
$$

Throughout this paper, $C\left(\mathbb{F}_{q}\right)$ denotes the set of $\mathbb{F}_{q}$-points of $C$, in other words, $C\left(\mathbb{F}_{q}\right)=C \cap \mathbb{P}^{r}\left(\mathbb{F}_{q}\right)$, where $\mathbb{P}^{r}\left(\mathbb{F}_{q}\right)$ is the set of $\mathbb{F}_{q}$-points of $\mathbb{P}^{r}$.

\section{Combinatorial approach}

We regard $\mathbb{P}^{r}\left(\mathbb{F}_{q}\right)$ as the $r$-dimensional finite projective space over $\mathbb{F}_{q}$.

Definition 2.1 Suppose $r \geq 2$. For a subset $X \subset \mathbb{P}^{r}\left(\mathbb{F}_{q}\right)$, the s-degree 2 of $X$ is the maximum number of points of $X$ that lie on a hyperplane of $\mathbb{P}^{r}\left(\mathbb{F}_{q}\right)$. The s-degree of $X$ is denoted by s-deg $X$.

The total number of points of $X$ is denoted by $N$. If $r=2$ and s-deg $X=$ $d, X$ is called an $(N, d)$-arc [1, (12.1)].

In the following lemma, $\lfloor\alpha\rfloor$ denotes the integer part of a real number $\alpha$.

Proposition 2.2 For $X \subset \mathbb{P}^{r}\left(\mathbb{F}_{q}\right)$ of s-degree $d$, the cardinality $N$ of $X$ is bounded by

$$
N \leq(d-1) q+1+\left\lfloor\frac{d-1}{q^{r-2}+q^{r-3}+\cdots+q+1}\right\rfloor .
$$

Proof. Fix a point $P_{0} \in X$. Let $\check{P}_{0}=\left\{H \in \check{\mathbb{P}}^{r}\left(\mathbb{F}_{q}\right) \mid P_{0} \in H\right\}$, where $\check{\mathbb{P}}^{r}\left(\mathbb{F}_{q}\right)$ denotes the set of hyperplanes of $\mathbb{P}^{r}\left(\mathbb{F}_{q}\right)$. Let

$$
\mathcal{P}=\left\{(P, H) \in\left(X \backslash\left\{P_{0}\right\}\right) \times \check{P}_{0} \mid P \in H\right\} .
$$

Moreover, $\pi_{1}: \mathcal{P} \rightarrow X \backslash\left\{P_{0}\right\}$ denotes the first projection and $\pi_{2}: \mathcal{P} \rightarrow \check{P}_{0}$ the second projection.

Let $P \in X \backslash\left\{P_{0}\right\}$. Since $\pi_{1}^{-1}(P)$ is the set of hyperplanes that contain the line $P_{0} P,{ }^{\#} \pi_{1}^{-1}(P)=q^{r-2}+q^{r-3}+\cdots+1$. Hence

$$
{ }^{\#} \mathcal{P}=\sum_{P \in X \backslash\left\{P_{0}\right\}}{ }^{\#} \pi_{1}^{-1}(P)=(N-1)\left(q^{r-2}+q^{r-3}+\cdots+1\right) .
$$

\footnotetext{
${ }^{1}$ As for the definition and the basic properties of order-sequence, see [2, 7.6]

${ }^{2}$ This jargon is an abbreviation for 'set-theoretic degree'. We want to reserve the simple terminology 'degree' for the degree of a curve.
} 
On the other hand, since s-deg $X=d,{ }^{\#}\left(H \cap\left(X \backslash\left\{P_{0}\right\}\right)\right) \leq d-1$ for any $H \in \check{P}_{0}$. Hence

$$
{ }^{\#} \mathcal{P} \leq(d-1)^{\#} \check{P}_{0}=(d-1)\left(q^{r-1}+q^{r-2}+\cdots+1\right) .
$$

Therefore

$$
\begin{aligned}
N & \leq(d-1) \frac{q^{r-1}+\cdots+1}{q^{r-2}+\cdots+1}+1 \\
& =(d-1) q+1+\frac{d-1}{q^{r-2}+\cdots+1} .
\end{aligned}
$$

This completes the proof.

Remark 2.3 When $r=2$, the bound (2) is rather trivial, that is, $N \leq$ $(d-1) q+d($ see $[1,(12.5)])$.

\section{$3 \quad$ Number of points of a nondegenerate irreducible curve}

In this section, we consider an irreducible curve $C$ in $\mathbb{P}^{r}$ with $r \geq 3$ defined over $\mathbb{F}_{q}$. Moreover we assume $C$ to be nondegenerate, that is, no hyperplane of $\mathbb{P}^{r}$ contains $C$. For a point $P \in C$ and a hyperplane $H$ of $\mathbb{P}^{r}$ with $H \ni P$, let $h$ be a local equation of $H$ around $P$. Under this situation, $V(h)$ denotes the hyperplane $H$. The intersection multiplicity $i(H . C ; P)$ of $C$ with $H$ at $P$ is

$$
i(H . C ; P)=\operatorname{dim} \mathcal{O}_{P, C} /(\bar{h}),
$$

where $\bar{h}$ is the image of $h$ in the local ring $\mathcal{O}_{P, C}$ of $P \in C$.

Lemma 3.1 For a point $P \in \mathbb{P}^{r}\left(\mathbb{F}_{q}\right)$,

$$
\sum_{H \in \check{P}}(i(H . C ; P)-1) \geq \frac{q^{r-1}+q^{r-2}+\cdots+q+1-r}{q-1},
$$

where $\check{P}$ is the set of $\mathbb{F}_{q}$-hyperplanes passing through $P$.

Proof. First suppose $P$ is a nonsingular point of $C$. Without loss of generality, we may assume that $P=(1,0, \ldots, 0)$. Let $x_{1}, \ldots, x_{r}$ be a system of affine coordinate functions around $P$ with $x_{1}(P)=\cdots=x_{r}(P)=0$, each of which is defined over $\mathbb{F}_{q}$. Then $\check{P}=\left\{V\left(\alpha_{1} x_{1}+\cdots+\alpha_{r} x_{r}\right) \mid\left(\alpha_{1}, \ldots, \alpha_{r}\right) \in\right.$ $\left.\mathbb{P}^{r-1}\left(\mathbb{F}_{q}\right)\right\}$. We choose a local parameter $t$ at $P \in C$ which is defined over $\mathbb{F}_{q}$. Through the identification $\widehat{\mathcal{O}}_{P, C}=\overline{\mathbb{F}}_{q}[[t]], x_{i}$ can be written as

$$
x_{i}=a_{i 1} t+a_{i 2} t^{2}+\cdots \quad(i=1,2, \ldots, r)
$$


in $\overline{\mathbb{F}}_{q}[[t]]$, where $a_{i j} \in \mathbb{F}_{q}$. Applying elementary row-operations over $\mathbb{F}_{q}$ to $\left(a_{i j}\right)_{\substack{i=1,2, \ldots, r \\ j=1,2, \ldots}}$, we have the following form:

$$
\left\{\begin{array}{cccccc}
x_{1}^{\prime} & = & t^{j_{1}}+ & \cdots & \cdots & \cdots \\
\vdots & & & & & \\
x_{i}^{\prime} & = & & t^{j_{i}}+ & \cdots & \cdots \\
\vdots & & & & & \\
x_{r}^{\prime} & = & & & t^{j_{r}}+ & \cdots
\end{array}\right.
$$

where $0<j_{1}=1<j_{2}<\cdots<j_{r}$ and the $\mathbb{F}_{q}$-vector space spanned by $x_{1}^{\prime}, \ldots, x_{r}^{\prime}$ is the original space spanned by $x_{1}, \ldots, x_{r}$. By using this renewed system of affine coordinate functions around $P$, we have a filtration $\check{P}=$ $V_{1} \supset V_{2} \supset V_{2} \supset \cdots \supset V_{r}$, where

$$
V_{i}=\left\{V\left(\alpha_{i} x_{i}^{\prime}+\cdots+\alpha_{r} x_{r}^{\prime}\right) \mid\left(\alpha_{i}, \ldots, \alpha_{r}\right) \in \mathbb{P}^{r-i}\left(\mathbb{F}_{q}\right)\right\} .
$$

If $H=V(h) \in V_{i} \backslash V_{i+1}$, then $h=\alpha_{i} t^{j_{i}}+\cdots$ with $\alpha_{i} \neq 0$. Hence $i(H . C ; P)=$ $j_{i} \geq i$. Therefore

$$
\begin{aligned}
\sum_{H \in \check{P}}(i(H . C ; P)-1) & \geq \sum_{i=1}^{r}(i-1) q^{r-i} \\
& =\frac{q^{r-1}+q^{r-2}+\cdots+1-r}{q-1} .
\end{aligned}
$$

Secondly, suppose $P$ is a singular point of $C$. Hence $i(H . C ; P) \geq 2$ for any $H \in \check{P}$. Therefore

$$
\begin{aligned}
\sum_{H \in \check{P}}(i(H . C ; P)-1) & \geq{ }^{\#} \check{P}=q^{r-1}+q^{r-2}+\cdots+1 \\
& >\frac{q^{r-1}+q^{r-2}+\cdots+1-r}{q-1} .
\end{aligned}
$$

This completes the proof.

Theorem 3.2 Let $C$ be a nondegenerate irreducible curve of degree $d$ in $\mathbb{P}^{r}$ over $\mathbb{F}_{q}$. Then

$$
N_{q}(C) \leq \frac{(q-1)\left(q^{r}+q^{r-1}+\cdots+1\right)}{q^{r}+q^{r-1}+\cdots+q-r} d=\frac{(q-1)\left(q^{r+1}-1\right)}{q\left(q^{r}-1\right)-r(q-1)} d .
$$

Proof. Let us consider the point-hyperplane correspondence with respect to $C$ over $\mathbb{F}_{q}$ :

$$
\mathcal{Q}:=\left\{(P, H) \in C\left(\mathbb{F}_{q}\right) \times \check{\mathbb{P}}^{r}\left(\mathbb{F}_{q}\right) \mid P \in H\right\}
$$


Let $\pi_{1}: \mathcal{Q} \rightarrow C\left(\mathbb{F}_{q}\right)$ and $\pi_{2}: \mathcal{Q} \rightarrow \check{\mathbb{P}}^{r}\left(\mathbb{F}_{q}\right)$ be the first and second projections respectively. If $H \in \pi_{2}(\mathcal{Q}) \subseteq \check{\mathbb{P}}^{r}\left(\mathbb{F}_{q}\right)$, then $\pi_{2}^{-1}(H)=\left(H \cap C\left(\mathbb{F}_{q}\right)\right) \times\{H\}$, and $d-\sum_{P \in H \cap C\left(\mathbb{F}_{q}\right)} i(H . C ; P) \geq d-(H . C)=0$. Hence

$$
\begin{aligned}
{ }^{\#} \pi_{2}^{-1}(H) & \leq \#\left(H \cap C\left(\mathbb{F}_{q}\right)\right)+d-\sum_{P \in H \cap C\left(\mathbb{F}_{q}\right)} i(H . C ; P) \\
& =d-\sum_{P \in H \cap C\left(\mathbb{F}_{q}\right)}(i(H . C ; P)-1) .
\end{aligned}
$$

Hence

$$
\begin{aligned}
{ }^{*} \mathcal{Q} & =\sum_{H \in \pi_{2}(\mathcal{Q})}{ }^{\#} \pi_{2}^{-1}(H) \\
& \leq \sum_{H \in \pi_{2}(\mathcal{Q})}\left(d-\sum_{P \in H \cap C\left(\mathbb{F}_{q}\right)}(i(H . C ; P)-1)\right) \\
& \leq d\left(q^{r}+q^{r-1}+\cdots+1\right)-\sum_{P \in C\left(\mathbb{F}_{q}\right)} \sum_{H \in \check{P}}(i(H . C ; P)-1)
\end{aligned}
$$

because ${ }^{\#} \pi_{2}(\mathcal{Q}) \leq q^{r}+q^{r-1}+\cdots+1$ and

$$
\mathcal{Q}=\bigsqcup_{H \in \pi_{2}(\mathcal{Q})}\left(\bigsqcup_{P \in H \cap C\left(\mathbb{F}_{q}\right)}\{(P, H)\}\right)=\bigsqcup_{P \in C\left(\mathbb{F}_{q}\right)}\left(\bigsqcup_{H \in \check{P}}\{(P, H)\}\right)
$$

Applying Lemma 3.1 to (3), we have

$$
{ }^{\#} \mathcal{Q} \leq d\left(q^{r}+q^{r-1}+\cdots+1\right)-\frac{q^{r-1}+q^{r-2}+\cdots+1-r}{q-1} N_{q}(C) .
$$

On the other hand,

$$
{ }^{\#} \mathcal{Q}=\sum_{P \in C\left(\mathbb{F}_{q}\right)}{ }^{\#} \pi_{1}^{-1}(P)=\left(q^{r-1}+q^{r-2}+\cdots+1\right) N_{q}(C) .
$$

From (4) and (5), we have the desired bound for $N_{q}(C)$.

Corollary 3.3 Under the same assumption as Theorem 3.2,

$$
N_{q}(C) \leq(q-1) d+\frac{r+1}{q^{r-1}+2 q^{r-2}+\cdots+(r-1) q+r} d .
$$

Proof. Let $N=N_{q}(C)$. By Theorem $\underline{3.2}$,

$$
\frac{q^{r}+q^{r-1}+\cdots+q-r}{q-1} N \leq d\left(q^{r}+q^{r-1}+\cdots+1\right) .
$$


Note that

$$
\begin{aligned}
q^{r}+q^{r-1}+\cdots+q-r & =\sum_{i=1}^{r}\left(q^{i}-1\right) \\
& =\sum_{i=1}^{r}(q-1)\left(q^{i-1}+q^{i-2}+\cdots+1\right) \\
& =(q-1)\left(\sum_{j=1}^{r} j q^{r-j}\right)
\end{aligned}
$$

Therefore, if we put $S=\sum_{j=1}^{r} j q^{r-j}$, then $S N \leq d((q-1) S+r+1)$. This completes the proof.

\section{Proof of Theorem 1.1 and Corollary 1.2}

Now we give proofs of the main theorem and its corollary.

Proof of Theorem 1.1. Let $L$ be the minimal linear subspace of $\mathbb{P}^{r}$ so that $L \supset C$. Since $C$ is defined over $\mathbb{F}_{q}, L \cap L^{(q)} \supset C$, where $L^{(q)}$ is the image of $L$ by the $q$-Frobenius map. By the minimality of $L, L=L^{(q)}$, that is, $L$ is an $\mathbb{F}_{q}$-space. Since $C$ is not contained any plane, $\operatorname{dim} L \geq 3$. Therefore we may assume that $C$ is nondegenerate in $\mathbb{P}^{r}$.

(i) Suppose $d \leq q^{r-2}+q^{r-3}+\cdots+1$. Since $\operatorname{deg} C=d$, the s-degree $d^{\prime}$ of $C\left(\mathbb{F}_{q}\right)$ is at most $d$. By the combinatorial bound (2) with our assumption,

$$
N_{q}(C) \leq\left(d^{\prime}-1\right) q+1 \leq(d-1) q+1 .
$$

(ii) Suppose $d \geq q$. In this case, we have $N_{q}(C) \leq(d-1) q+1$ by Corollary 3.3. In fact,

$$
\begin{aligned}
& (d-1) q+1-\left((q-1) d+\frac{r+1}{q^{r-1}+2 q^{r-2}+\cdots+(r-1) q+r} d\right) \\
= & \left(1-\frac{r+1}{q^{r-1}+2 q^{r-2}+\cdots+(r-1) q+r}\right) d-q+1 .
\end{aligned}
$$

Since the coefficient of $d$ is positive and $d \geq q$,

$$
\text { the quantity (6) } \geq 1-\frac{r+1}{q^{r-1}+2 q^{r-2}+\cdots+(r-1) q+r} q .
$$

Since $r \geq 3$,

$$
\begin{aligned}
& \left(q^{r-1}+2 q^{r-2}+\cdots+(r-1) q+r\right)-(r+1) q= \\
& q^{r-1}+\cdots+(r-3) q^{3}+(r-3) q^{2}+(q-1)^{2}+(r-1)>0 .
\end{aligned}
$$

Hence (77) is positive. 
Obviously, $q<q^{r-2}+q^{r-3}+\cdots+1$ because $r \geq 3$. Hence (i) and (ii) imply the desired bound.

Proof of Corollary 1.2, If $r=2$, this is nothing but the main theorem of [5]. So we assume that $r \geq 3$.

(i) First we show that we may assume $C$ to be irreducible over $\mathbb{F}_{q}$. Let $C=C_{1} \cup \cdots \cup C_{s}$ be the decomposition of $C$ into $\mathbb{F}_{q}$-irreducible components, and $\operatorname{deg} C_{i}=d_{i}(i=1, \ldots, s)$. If $N_{q}\left(C_{i}\right) \leq\left(d_{i}-1\right) q+1$ holds true for any $C_{i}$, then

$$
\begin{aligned}
N_{q}(C) & \leq \sum_{i=1}^{s} N_{q}\left(C_{i}\right) \leq \sum_{i=1}^{s}\left(\left(d_{i}-1\right) q+1\right) \\
& =(d-s) q+s<(d-1) q+1 .
\end{aligned}
$$

When $q=4$ and $s \geq 2$, suppose each of the first $s^{\prime}$ components $C_{1}, \ldots, C_{s^{\prime}}$ is contained in a plane and isomorphic to $K$ over $\mathbb{F}_{4}$, and the remaining $s-s^{\prime}$ components are not. Then $d_{1}=\cdots=d_{s^{\prime}}=4, d=4 s^{\prime}+\sum_{i=s^{\prime}+1}^{s} d_{i}$ and $N_{q}\left(C_{1}\right)=\cdots=N_{q}\left(C_{s^{\prime}}\right)=14$. Hence

$$
\begin{aligned}
N_{q}(C) & \leq 14 s^{\prime}+\sum_{i=s^{\prime}+1}^{s}\left(\left(d_{i}-1\right) 4+1\right) \\
& =(d-1) 4+1+3-3 s+s^{\prime} \\
& \leq(d-1) 4+1+3-2 s \quad\left(\text { because } s^{\prime} \leq s\right) \\
& <(d-1) 4+1 \quad \text { (because } s \geq 2) .
\end{aligned}
$$

(ii) Suppose $C$ is not absolutely irreducible. As the preliminary step of the proof of Theorem 1.1, we may assume that $C$ is nondegenerate in $\mathbb{P}^{r}$. Let $D$ be an irreducible component of $C$. Then $C=D \cup D^{(q)} \cup \ldots D^{\left(q^{t-1}\right)}$ for some $t \geq 2$, because $C$ is irreducible over $\mathbb{F}_{q}$. Hence $\operatorname{deg} D=\frac{d}{t} \leq \frac{d}{2}$, and $C\left(\mathbb{F}_{q}\right) \subset D \cap D^{(q)} \cap \ldots D^{\left(q^{t-1}\right)}$. When $C\left(\mathbb{F}_{q}\right)$ does not span $\mathbb{P}^{r}$, choose a hyperplane $H$ over $\mathbb{F}_{q}$ such that $H \supset C\left(\mathbb{F}_{q}\right)$. Since $C$ is nondegenerate, $H$ does not contain any components of $C$ because $H$ is defined over $\mathbb{F}_{q}$. Hence we have

$$
N_{q}(C) \leq(D . H)=\operatorname{deg} D \leq \frac{d}{2}<(d-1) q+1,
$$

which is the desired bound. Therefore we may assume that $C\left(\mathbb{F}_{q}\right)$ spans $\mathbb{P}^{r}$. Hence we can pick up $r-1$ points $Q_{1}, \ldots, Q_{r-1} \in C\left(\mathbb{F}_{q}\right)$ such that the linear space $L_{0}$ spanned by these $r-1$ points is an $\mathbb{F}_{q}$-linear subspace of codimension 2. Put $\#\left(L_{0} \cap C\left(\mathbb{F}_{q}\right)\right)=r^{\prime}$. Obviously $r^{\prime} \geq r-1$. Let $\left\{H_{0}, \ldots, H_{q}\right\}$ be the set of $\mathbb{F}_{q}$-hyperplanes, each of which contains $L_{0}$. Since $C\left(\mathbb{F}_{q}\right) \backslash L_{0} \subset \cup_{i=0}^{q}\left(D \cap H_{i} \backslash L_{0}\right)$,

$$
N_{q}(C) \leq\left(\frac{d}{t}-r^{\prime}\right)(q+1)+r^{\prime} \leq\left(\frac{d}{2}-r^{\prime}\right)(q+1)+r^{\prime} .
$$


Since

$$
(d-1) q+1-\left(\left(\frac{d}{2}-r^{\prime}\right)(q+1)+r^{\prime}\right)=r^{\prime} q+\left(\frac{d}{2}-1\right)(q-1)>0,
$$

(8) is bounded by $(d-1) q+1$.

Therefore we may assume that $C$ is absolutely irreducible, which is the case we already considered in Theorem 1.1.

\section{Asymptotic behavior}

In this section, we introduce an analogue of Ihara's constant $3(q)$.

Notation 5.1 $\tilde{\mathcal{C}}_{d}^{i}\left(\mathbb{F}_{q}\right)$ denotes the set of irreducible curve over $\mathbb{F}_{q}$ of degree $d$ in a projective space of some dimensions.

Remark 5.2 The set $\tilde{\mathcal{C}}_{d}^{i}\left(\mathbb{F}_{q}\right)$ consists of finitely many elements. In fact, any member $C \in \tilde{\mathcal{C}}_{d}^{i}\left(\mathbb{F}_{q}\right)$ can be embedded into $\mathbb{P}^{s}$ with $s \leq d$ over $\mathbb{F}_{q}$ as a degree $d$ curve.

Definition 5.3 Let $\tilde{M}_{q}^{i}(d):=\max \left\{N_{q}(C) \mid C \in \tilde{\mathcal{C}}_{d}^{i}\left(\mathbb{F}_{q}\right)\right\}$, which makes sense because of the finiteness of $\tilde{\mathcal{C}}_{d}^{i}\left(\mathbb{F}_{q}\right)$. The quantity

$$
D(q):=\limsup _{d \rightarrow \infty} \tilde{M}_{q}^{i}(d) / d
$$

measures the asymptotic behavior of $\tilde{M}_{q}^{i}(d)$.

We don't know yet the exact value of $D(q)$ for any $q$. Here we state just an observation.

\section{Proposition 5.4}

$$
\frac{1}{2} A(q) \leq D(q) \leq q
$$

Proof. Since $\tilde{M}_{q}^{i}(d) \leq(d-1) q+1$ by Theorem 1.1, we have $D(q) \leq q$. Let $N_{q}(g)$ be the maximum number of $\mathbb{F}_{q}$-points on a nonsingular curve of genus $g$. By definition, $A(q)=\lim \sup _{g \rightarrow \infty} N_{q}(g) / g$. It is known that $A(q)>0$ by Serre (for more and precise information on the Ihara's constant, see [9, Chap. 3]). Hence, for most $g$ 's, $N_{q}(g)$ is achieved by a nonhyperelliptic curve, which can be embedded into $\mathbb{P}^{g-1}$ over $\mathbb{F}_{q}$ as a degree $2 g-2$ curve. Therefore

$$
\limsup _{g \rightarrow \infty} N_{q}(g) /(2 g-2)=\frac{1}{2} A(g)
$$

is a lower bound for $D(g)$.

\footnotetext{
${ }^{3}$ We use this terminology after [6, 7.1.1].
} 


\section{References}

[1] J. W. P. Hirschfeld, Projective geometries over finite fields (second edition), Oxford University Press, Oxford, 1998.

[2] J. W. P. Hirschfeld, G. Korchmáros and F. Torres, Algebraic curves over a finite field, Princeton Univ. Press, Princeton and Oxford, 2008.

[3] M. Homma and S. J. Kim, Around Sziklai's conjecture on the number of points of a plane curve over a finite field, Finite Fields Appl. 15 (2009), 468-474.

[4] M. Homma and S. J. Kim, Sziklai's conjecture on the number of points of a plane curve over a finite field II, in: G. McGuire, G.L. Mullen, D. Panario, I.E. Shparlinski (Eds.), Finite Fields: Theory and Applications, in: Contemp. Math., vol. 518, AMS, Providence, 2010, 225-234.

[5] M. Homma and S. J. Kim, Sziklai's conjecture on the number of points of a plane curve over a finite field III, Finite Fields Appl. 16 (2010) 315-319.

[6] H. Stichtenoth, Algebraic function fields and codes (second edition), GTM 254, Springer-Verlag, Berlin and Heidelberg, 2008.

[7] K.-O. Stöhr and J. F. Voloch, Weierstrass points and curves over finite fields, Proc. London Math. Soc. (3) 52 (1986) 1-19.

[8] P. Sziklai, A bound on the number of points of a plane curve, Finite Fields Appl. 14 (2008) 41-43.

[9] M. Tsfasman, S. Vlăduţ and D. Nogin, Algebraic geometric codes: Basic notions, SURV 139, AMS, Providence, 2007. 